\title{
Diferenças de Sexo e Escolaridade na Manifestação de Stress em Adultos Jovens
}

\author{
Sandra Leal Calais ${ }^{12}$ \\ Universidade Estadual Paulista/Bauru \\ Livia Márcia Batista de Andrade \\ Marilda Emmanuel Novaes Lipp \\ Pontifícia Universidade Católica de Campinas
}

\begin{abstract}
Resumo
Este estudo pesquisou sintomas de stress em adultos jovens, relacionando-os com o sexo e ano escolar em curso. Investigou também o tipo e a freqüência de sintomas. Participaram 295 estudantes de 15 a 28 anos, sendo 150 mulheres e 145 homens, que cursavam o primeiro e terceiro anos do ensino médio, curso pré-vestibular e primeiro e quarto anos de ensino superior. A avaliação do stress foi realizada através do Inventário de Sintomas de Stress de Lipp. Os resultados acusaram correlação significativa entre sexo e nível de stress $(p<0,0001)$ sendo que as mulheres apresentaram maior nível de stress em todos os grupos avaliados. Maior índice de stress surgiu em estudantes do curso Pré-Vestibular, seguidos do terceiro ano do ensino médio. A sintomatologia apresentada foi predominantemente psicológica e os sintomas mais prevalentes foram sensibilidade emotiva excessiva, para as mulheres e, para os homens, pensamento recorrente.

Palavras-chave: Stress; gênero; grau de escolaridade.
\end{abstract}

Gender and Schooling Differences in Stress Symptoms in Young Adults

\begin{abstract}
Gender differences in stress symptoms have been a topic of recent interest. This study investigated stress symptoms in young adults in relation to sex and school year. One hundred and fifty female and 145 male students, age 15 to 28 , were the participants. They were recruited from freshman and senior high school classes, preparatory school for college, and the first and fourth year of a college in São Paulo. The Lipp Stress Symptom Inventory was used to evaluate self perceived stress. Results indicated a high correlation between gender and level of stress $(p<0,0001)$. Women compared to men had more symptoms. Students in the preparatoy school class had the highest incidence of stress, followed by high school seniors. Students had a higher number of psychological symptoms and the most frequently mentioned were excessive emotional sensitivity for the female participants, and recurrent thoughts for the male students.

Keywords: Stress; gender; age.
\end{abstract}

O stress é uma reação intensa do organismo frente a qualquer evento bom ou mau que altere a vida do indivíduo. Essa reação ocorre, em geral, frente à necessidade de adaptação exigida do indivíduo em momentos de mudança (Everly, 1989). Sendo o Brasil um país em desenvolvimento onde as mudanças sociais, morais, econômicas e tecnológicas ocorrem com uma rapidez muito grande, é de se prever que o nível de stress do brasileiro seja significativo. A prevalência do stress provavelmente é bastante elevada no Brasil, pois chega a 32\% em São Paulo, como verificado por Lipp, Pereira, Floksztrumpf, Muniz e Ismael (1996) em uma amostra de 1818 pessoas que transitavam em um aeroporto nesta cidade.

\footnotetext{
${ }^{1}$ Endereço para correspondência: Rua João Andreoli, 2103, 17018 090, Bauru, SP. Fone: (14) 32342888.E-mail: scalais@fc.unesp.br / mscalais@terra.com.br ${ }^{2}$ Apoio Capes.
}

O processo de stress passa por três fases: alerta, resistência e exaustão. A fase de alerta se caracteriza por reações do sistema nervoso simpático, quando o organismo percebe o estressor (o evento). A resistência apresenta-se quando esse estressor permanece presente por períodos prolongados ou se é de grande dimensão. Na fase de exaustão, o stress ultrapassou a possibilidade do indivíduo conviver com ele e está associado a diversos problemas como úlceras, gengivites, psoríase, hipertensão arterial, depressão, ansiedade, problemas sexuais, dentre outros. Cada fase envolve uma sintomatologia diferenciada que é acompanhada de mudanças hormonais correspondentes (Selye, 1956).

Embora a maioria dos estudos na área se preocupe mais com o stress na população de adultos, alguns trabalhos têm sido realizados enfocando o stress infantil (Anthony \& Cohler, 1992; Lipp, 2000; Mulatu, 1995; Tricoli, 1997; Vilela, 1996). Por exemplo, Mulatu (1995) em um estudo 
com crianças de 6 a 11 anos da Etiópia, pesquisou 317 meninas e 294 meninos, para verificar a prevalência de fatores psicopatológicos nessas crianças, gerada pela situação de carência vivida pelo país. O estudo revelou que, em ambas as amostras, existiam problemas como: agressividade, incomunicabilidade, ansiedade, depressão, hiperatividade, imaturidade e delinqüência. A presença dos sintomas de stress foi verificada em $21,45 \%$ dos meninos e $25,17 \%$ das meninas. A maior prevalência do stress em meninas, encontrada por Mulatu (1995) aponta para uma realidade que merece ser aprofundada.

Poucos estudos têm se dedicado à investigação do stress no adolescente e no adulto jovem. Curiosamente, Arnett (1999) considera que apesar de que nem todo adolescente tem stress, a probabilidade de desenvolvê-lo é maior na adolescência do que em qualquer outra faixa etária, dependendo da cultura e de diferenças individuais existentes.

Mesmo sem utilizar a ótica do stress, autores como Knobel (1970/1985) caracterizam a adolescência por períodos de extrema instabilidade. Na cultura brasileira, o adolescente apresenta crises religiosas, conflitos familiares, dificuldades sexuais, "uma síndrome normal da adolescência". Não podemos ignorar o elemento sócio-cultural nessas manifestações da adolescência, mas deve ser também levado em conta o embasamento psicobiológico que lhe dá características universais.

Schowalter (1995) define adolescência como o período de transição do apoio integral dos pais à auto-suficiência e alerta que o seu princípio e o seu fim variam grandemente. É mais exato conceber a adolescência levando em consideração o desenvolvimento físico, psicológico e social. Esse autor pesquisa o que ocorre na adolescência tardia, verificando a transição do período escolar e decisões futuras profissionais. Para ele, pesquisas nesta área são importantes, pois ainda há muito para ser aprendido sobre as alterações fisiológicas da puberdade e seus efeitos sobre o comportamento e a psicopatologia visto que as meninas parecem ter uma diminuição da auto-estima, um aumento da preocupação com a imagem corporal e um aumento de transtornos alimentares e depressão clínica, que não estão presentes nos meninos. Para ambos os sexos, há um aumento dos transtornos bipolares e esquizofrenia. Os meninos mostram um grande aumento em homicídios, suicídios e comportamento anti-social. Embora estes transtornos e comportamentos não sejam exclusivos da adolescência, parece ser essa a idade mais propícia para esclarecer suas origens biopsicossociais. Diferenças sexuais nessa etapa do desenvolvimento refletem-se em conflitos próprios podendo até determinar psicopatologias específicas.

Considerando-se que o adolescente e o jovem adulto se constituem em uma população suscetível e influenciável às estimulações externas psicossociais, conhecer como o stress se manifesta neste grupo é fundamental para uma futura elaboração de métodos eficazes de seu manejo. Além disto, devido ao fato de que o stress se manifesta tanto no psicológico como no físico, nem sempre é fácil o diagnóstico diferencial com patologias físicas e mentais.

Encontram-se diferenças significativas entre os sexos quanto ao stress, porém essas diferenças necessitam ser analisadas sistematicamente uma vez que para a prevenção dos efeitos negativos do stress no funcionamento humano torna-se importante averiguar diferenças de sexo relacionadas com idade e escolaridade dos jovens. Esta é uma área ainda nova e controvertida na literatura.

A indicação de que mulheres talvez experienciem mais stress do que os homens vem de várias fontes. Por exemplo, uma pesquisa realizada por Gadzella, Ginther, Tomcala e Bryant (1991), utilizando a Tennessee Stress Scale-L (McWilliams \& Schnorr, 1986), encontrou diferenças quanto ao stress em profissionais de negócios em função de sexo, idade, situação conjugal, tipo de trabalho, ter filhos e possuir animais de estimação. Os resultados mostram que mulheres apresentam escores mais altos que os homens em toda a escala (Tennessee Stress Scale-L; McWilliams \& Schnorr, 1986). Escores para mulheres foram significantemente mais altos em produção, sintomas e no total de stress. Os autores referiram que as mulheres avaliadas relataram mais situações estressantes do que homens.

Scott (1992) avaliou o stress entre administradores escolares de ambos os sexos, no trabalho e em seus lares. As mulheres se apresentaram significativamente mais estressadas que os homens tanto em escolas públicas como privadas e em seus lares. Nos lares com criança, o stress era maior do que nos sem crianças e em pequenas escolas (1000 ou menos estudantes) mais do que em grandes escolas (20000 ou mais estudantes). No entanto, esse autor comenta que estes dados não podem comprovar efetivamente que as mulheres são mais estressadas do que os homens, mas que podem ser mais aptas para lamentarem e expressarem seus sentimentos e serem mais envolvidas pela vida de outras pessoas do que os homens.

Há autores que enfocam diferenças de sexo do ponto de vista biológico favorecendo o desenvolvimento de psicopatologias em homens e mulheres. Por exemplo, Seeman (1997) enfoca que estrogênios são neuroproteínas ligadas à degeneração neural, crescimento e suscetibilidade para toxinas. Estrogênios, notavelmente estradiol, possuem um papel ativo na organização do desenvolvimento cerebral e um papel crucial na manutenção da atividade cerebral. Entre outras funções, eles protegem neurônios contra disfunções de desenvolvimento (esquizofrenia) e disfunções degenerativas (Doença de Alzheimer). As flutuações cíclicas 
de estrogênio e progesterona aumentam as respostas de stress, as quais conferem suscetibilidade para depressão e ansiedade.

Há muitas razões para se acreditar que o desenvolvimento evolutivo comporte uma neuroproteção especial, mas também uma sensibilidade ao stress mediante o papel dos hormônios femininos (especialmente por sua ciclicidade), os quais conseqüentemente conferem vantagens e desvantagens para as mulheres. Durante os anos de juventude, as mulheres parecem estar comparativamente mais protegidas de doenças psicóticas severas e mais vulneráveis que homens para depressão e ansiedade.

As mulheres apresentam, mais que os homens, quadros de estados de ansiedade também para Spielberger (1976), existindo alguma indicação de que elas tornam-se mais ansiosas devido ao nível de circulação de estrogênio e progesterona. Isto poderia ser responsável por mulheres, como um grupo, serem mais sensíveis que homens para os efeitos ansiógenos de stress não específico.

Apesar de anteriormente sustentarem que as mulheres são geralmente mais vulneráveis que homens para estressores, recentes pesquisas indicam que diferenças sexuais na vulnerabilidade são altamente específicas e dependem do tipo de estressor e desordem envolvidos.

Lipp e colaboradores (1996) afirmam que para se prevenir e tratar o stress adequadamente existe considerável interesse em se saber se homens e mulheres diferem no nível de stress que apresentam e no modo como ele se manifesta, se na área somática ou cognitiva. Nesse estudo, a amostra espontaneamente se constituiu de 56\% de homens e $44 \%$ de mulheres. A faixa etária prevalente era a de mais de 46 anos. Verificou-se haver uma diferença significativa no número de homens e mulheres com stress no momento, uma vez que $19 \%$ das mulheres demonstraram sintomas de stress em comparação com 13\% dos homens. Foi levantada a hipótese de que é possível que as mulheres do estudo estivessem no período da menopausa, o que poderia estar contribuindo para o seu alto nível de stress. Outros estudos se fazem necessários para comparar as diversas faixas etárias a fim de se controlar o efeito da menopausa e averiguar se em outras idades homens e mulheres diferem quanto à vulnerabilidade ao stress.

A escola, com todas as suas expectativas e exigências, pode ser também uma grande fonte de estressores. Para Langston e Cantor (1989) deve-se considerar que a transição na vida acadêmica dos estudantes no início de seus estudos universitários pode gerar um aumento de responsabilidade, ansiedade e competitividade, o que facilitaria o stress. Além das mudanças próprias de ensino, os alunos se deparam com as incertezas naturais da escolha profissional. Fisher (1994), em seus estudos com universitários, também verificou a ocorrência de stress na época de transição para a universidade, decorrente da mudança de planos (novas etapas), tarefas acadêmicas, dificuldades financeiras e, sobretudo, sociais que marcam a vida dos universitários.

Campos, Rocha, Campos e Oliveira (1996), avaliaram um grupo de primeiranistas de uma instituição de ensino superior do estado de São Paulo durante o ano letivo. Os resultados deste estudo sugerem que os comportamentos dos sujeitos variam de acordo com o curso escolhido (medicina, engenharia e psicologia). Verificou-se a tendência de diminuição do reajustamento social nos alunos de medicina, ao contrário dos alunos de engenharia e psicologia que, indicaram um aumento do reajuste social, sugerindo fontes de stress diretamente relacionadas com a vida acadêmica.

Outro trabalho realizado (Haslam, Stevens \& Haslam, 1989) relata que os principais problemas demonstrados por estudantes universitários são a depressão, abuso de drogas e, no caso das estudantes de sexo feminino, distúrbios de alimentação. Esta problemática parece ser relacionada ao nível de stress apresentado pelo indivíduo.

Deste modo, saber como o stress atua no jovem, que sintomas são mais prevalentes no sexo masculino comparado com o feminino, como os sintomas diferem dependendo da escolaridade é de importância para todos os profissionais que atuam com adolescentes.

O presente trabalho visou a investigar sintomas de stress em adultos jovens, relacionando-os com o sexo e ano escolar em curso. Investigou também o tipo de prevalência de sintomas, se físicos ou psicológicos, e quais os sintomas mais freqüentemente encontrados em homens e mulheres jovens.

\section{Método}

\section{Participantes}

Duzentos e noventa e cinco jovens, sendo 145 do sexo masculino e 150 do sexo feminino participaram do estudo. Os participantes foram divididos em cinco grupos de cerca de 60 sujeitos, metade de cada sexo, por grau de escolaridade sendo: G1 - primeiro ano do ensino médio, G2- terceiro ano do ensino médio, G3- curso prévestibular, G4- primeiro ano do ensino superior, G5quarto ano do ensino superior. A faixa etária do grupo total variou de 15 a 28 anos, com idade média de 18,3 anos. A idade média do G1 foi 15,5 anos e do G2 foi 17,5 anos. O G3 apresentou a idade média de 18,1 anos e o G4 a de 19 anos. Já o G5 possuía uma idade média de 22 anos. A pesquisa foi realizada em três colégios de Campinas, interior de São Paulo (G1 e G2), em um curso pré-vestibular da cidade de Bauru, SP(G3) e em 
uma instituição de Ensino Superior, curso de Odontologia, também em Campinas (G4 e G5). As escolas e curso superior foram da rede particular de ensino. $O$ curso de Odontologia foi escolhido por ser um curso com procura equivalente de homens e mulheres.

\section{Material}

$\mathrm{Na}$ avaliação do stress foi utilizado o Inventário de Sintomas de Stress de Lipp (ISS) já validado para sujeitos a partir de 15 anos (Lipp \& Guevara, 1994).

O ISS se constitui de uma lista de sintomas físicos (Ex.: boca seca, tensão muscular, formigamento das extremidades) e psicológicos (Ex.: dúvida quanto a si mesmo, aumento súbito de motivação, perda do senso de humor) divididos em três quadros. Baseia-se no modelo trifásico de Selye (1956) sendo que cada quadro corresponde a uma das fases do modelo. $O$ respondente deve indicar primeiro quais os sintomas do primeiro quadro que experienciou nas últimas 24 horas. A seguir deve assinalar que sintomas sentiu na última semana dentre os apresentados no quadro $2 \mathrm{e}$ finalmente deve assinalar, dentre os sintomas físicos e psicológicos do quadro 3 , quais experienciou no último mês. O ISS permite diagnosticar se a pessoa tem stress, em que fase do processo se encontra (alerta, resistência e exaustão) e se sua sintomatologia é mais típica da área somática ou cognitiva.

\section{Procedimentos}

Após a autorização das escolas, foi solicitada a autorização dos pais, quando o participante era menor de idade. Após a assinatura do termo de consentimento próprio ou dos pais, o Inventário de Sintomas de Stress foi aplicado nos primeiro e terceiro anos do ensino médio, curso pré-vestibular, primeiro e último ano da Faculdade de Odontologia, em uma única vez e na própria sala de aula.

Nas classes onde havia mais do que o número desejado de participantes, procedeu-se a uma seleção aleatória, pelos números pares das listas de presença, completando dois grupos de 30 alunos do sexo feminino e 30 alunos do sexo masculino de cada série selecionada. Os estudantes foram informados da não obrigatoriedade e assegurados quanto ao sigilo e demais práticas éticas.

\section{Resultados}

Dos 295 participantes, 65,60\% apresentavam stress, conforme os critérios do ISS de Lipp, havendo uma diferença entre homens e mulheres. Esses critérios são: apresentar mais de 6 dos 15 sintomas apresentados para as 24 horas, ou mais que 3 dos 15 sintomas para a última semana ou ainda mais que 8 dos 23 sintomas no último mês, conforme as questões (cognitivas e somáticas) apresentadas para cada período. Verificou-se que 79,30\% das mulheres possuíam sintomas significativos de stress enquanto que nos participantes do sexo masculino a percentagem com sintomas de stress era $51,72 \%$. O teste do Qui Quadrado revelou uma associação significativa $(\chi 2=23,75, p=0,0001)$ entre sexo e nível de stress, mostrando serem as mulheres mais estressadas na amostra global.

Conforme a Tabela 1, os alunos do curso pré-vestibular (G3) apresentavam o nível de stress mais alto e os do primeiro ano de ensino superior apresentavam o menor índice. $\mathrm{O}$ teste do qui-quadrado aplicado mostrou haver uma associação significativa entre stress e série sendo cursada pelos estudantes $(\chi 2=12,04, p=0,01)$. A comparação entre pares de grupos, realizada com o Teste Exato de Fisher, revelou a existência de uma associação significativa entre o grupo G3, que cursava o pré-vestibular, e grupo G1, que cursava o primeiro ano do ensino médio, $(p=0,004)$, sendo o stress mais elevado no G3. A associação foi também significativa entre o grupo G4, cujos integrantes estavam cursando o primeiro ano do ensino superior e o G3. A associação entre stress e nível escolar não foi significativa para os outros grupos estudados.

Tabela 1

Percentagens de Estudantes com Stress por Grupo e no Todo

\begin{tabular}{cc}
\hline Grupo & $\%$ \\
\hline 1 & 58,00 \\
2 & 70,00 \\
3 & 83,00 \\
4 & 57,00 \\
5 & 60,00 \\
Geral & 65,60
\end{tabular}

Para cada grupo, há diferenças, segundo o sexo, na prevalência do stress como pode ser visto na Tabela 2. Aplicando-se o Teste Exato de Fisher para verificar a significância da associação entre sexo e série escolar em curso, apurou-se que no G1 $(p=0,001)$ e no G5 $(p=0,01)$ havia esta associação, porém ela não atingiu o nível de significância no G2 $(p=0,15)$, no G3 $(p=0,29)$ e G4 $(p=0,06)$ mostrando que nestas séries não havia diferença significativa no nível de stress dos entrevistados devido ao sexo. Pode-se inferir que o sexo se constitui em um fator de risco para o stress no G1 (primeiro ano ensino médio) e no G5 (quarto ano do ensino superior).

Quanto às fases do stress, verificou-se que 6,19\% do grupo total se encontrava na fase de alerta, $92,78 \%$ em resistência e $1,03 \%$ na fase de exaustão mostrando uma grande incidência na segunda fase do stress. No que se refere às mulheres, 
Tabela 2

Percentagens de Estudantes com Stress por Sexo e Grupo

\begin{tabular}{ccc}
\hline Grupo & Mulheres $\%$ & Homens $\%$ \\
\hline 1 & 40,00 & 18,00 \\
2 & 40,00 & 30,00 \\
3 & 45,00 & 38,00 \\
4 & 35,00 & 22,00 \\
5 & 42,00 & 18,00 \\
\hline
\end{tabular}

$98,31 \%$ das que tinham stress se encontravam em resistência, $0,84 \%$ em alerta e exaustão igualmente. Os homens que tinham stress apresentavam-se com $84,00 \%$ na fase de resistência, $14,67 \%$ de alerta e $1,33 \%$ de exaustão.

Encontrou-se na amostra geral a predominância de sintomas psicológicos, sendo que $55,70 \%$ dos avaliados apresentavam este tipo de sintomatologia em maior grau. Os que tinham mais sintomas físicos se constituíam em $32,40 \%$ e $11,90 \%$ da amostra possuíam a presença de ambos com igual freqüência. Pode-se observar a prevalência dos sintomas segundo o ISS de Lipp na Tabela 3. O sintoma mais mencionado pelas mulheres foi a sensibilidade emotiva exagerada, seguido de irritabilidade excessiva, sensação de desgaste físico constante e cansaço constante. Para os homens, o sintoma mais presente foi o pensamento constante sobre um só assunto, seguido da sensação de desgaste físico constante e problemas com a memória. Deste modo, tanto homens como mulheres mostraram que a sensação de desgaste físico constante é muito freqüente na pessoa estressada.

Tabela 3

Prevalência de Sintomas da Fase Resistência por Sexo em \%

\begin{tabular}{lcc}
\hline Sintomas & Mulheres & Homens \\
\hline Esquecimento & 51,30 & 32,40 \\
Mal estar & 38,00 & 13,30 \\
Formigamento & 13,30 & 18,60 \\
Desgaste & 53,30 & 33,30 \\
Mudança apetite & 51,30 & 19,30 \\
Probl. dermatológico & 30,60 & 15,30 \\
Cansaço & 53,30 & 27,30 \\
Gastrite & 25,30 & 14,60 \\
Tontura & 37,30 & 15,30 \\
Emotividade & 68,00 & 21,30 \\
Dúvidas & 46,00 & 24,60 \\
Pensar recorrente & 46,60 & 36,60 \\
Irritação & 54,00 & 28,00 \\
Diminuição libido & 7,30 & 2,60 \\
\hline
\end{tabular}

Os sintomas que menos se apresentaram nos homens e nas mulheres foram igualmente diminuição da libido, mal estar generalizado, formigamento das extremidades e gastrite prolongada.

\section{Discussão}

Verificou-se a predominância de stress no sexo feminino, evidenciado pelo fato das mulheres apresentarem sintomas significativos de stress, em comparação com o sexo masculino. Estes dados suportam a pesquisa de Lipp e colaboradores (1996) que demonstrou em uma amostra de 1818 brasileiros que mais mulheres do que homens tinham sintomas de stress. Apóia também os resultados da investigação realizada por Tricoli (1997) mostrando uma maior incidência de stress em meninas de sete anos de idade quando comparadas com meninos da mesma faixa etária. Adicionalmente, os dados da presente pesquisa vão ao encontro dos de Tanganelli e Lipp (1998) que revelaram, em uma amostra de escolares da rede pública do Ensino Fundamental, uma percentagem maior de meninas estressadas do que seus colegas de classe do sexo masculino.

Até certo ponto estes dados são inesperados, pois considerando-se a cultura brasileira, onde o homem ainda é considerado o cabeça do casal e responsável pela manutenção financeira da familia, poderia se levantar a hipótese de que o stress seria maior no sexo masculino, ou pelo menos igual ao das mulheres. Na verdade, somente nos últimos anos é que se começou a encontrar uma maior incidência de stress em mulheres, talvez devido ao fato de que as investigações clínicas do stress, normalmente associado à vida adulta e profissional, 
referiam-se a profissionais com cargos de responsabilidade, tais como os executivos, policiais militares e controladores de tráfego aéreo, profissões estas onde há um predomínio do sexo masculino. Assim, a prevalência de sexo masculino era enfocada e não havia uma busca sistemática de diferenças entre homens e mulheres.

A pesquisa de Lipp e colaboradores (1996), utilizando uma população não clínica de pessoas que estavam aguardando vôo ou transitavam em um shopping center em São Paulo, acidentalmente, encontrou uma diferença significativa entre os sexos. A partir desta descoberta acidental, outros estudos estão averiguando uma possível diferença entre os sexos em diversas faixas etárias. Além da hipótese de que só agora se esteja começando a estudar estas diferenças e por isto os dados apresentem uma certa surpresa, há de se levantar a hipótese de que atualmente o nível de stress em mulheres esteja de fato aumentando.

Esta hipótese poderia se basear no fato de que no momento a sociedade exige das mulheres, de maneira geral, uma sobrecarga de atividades, onde a carreira (profissional ou acadêmica) é acrescida das exigências pessoais, biológicas, hormonais, sexuais e sociais. Evidência a este favor é fornecida pelo registro de mulheres com problemas cardiovasculares, que até algumas décadas atrás não existia. Lipp e Rocha (1994) comentam que nas doenças coronárias os homens são mais afetados que as mulheres até os 60 anos, quando as mulheres passam a superar os homens em prevalência de derrame cerebral. Independentemente da razão pela qual a mulher está tendo mais stress, este dado é de grande relevância não só para as próprias mulheres, mas também para a família e para a sociedade, no geral. Considerando-se que pesquisas mostram a importância da saúde mental da mãe para o bom funcionamento dos filhos (Earls, Beardslee \& Garrison, 1992; Short \& Johnston, 1997) e, levando em consideração, que o stress excessivo causa sensação de desgaste físico constante, irritabilidade, hipersensibilidade, sensação de alienação emocional e dificuldades com a memória, é de se entender a importância de se concentrar uma atenção especial às medidas preventivas e ao ensino de estratégias de enfrentamento que capacitem as mulheres a lidar melhor com o stress emocional ao qual estão sujeitas.

O dado observado neste trabalho de que um número tão alto de jovens estivesse na fase de resistência do stress é preocupante. Esta fase é conceituada por Selye (1956) como aquela em que a pessoa automaticamente utiliza suas reservas de energia para se reequilibrar, ou seja, nela ocorre uma ação reparadora do organismo tentando restabelecer o equilibrio interno. Nesta fase, dois sintomas que muitas vezes passam despercebidos ao clínico, aparecem de modo bastante freqüente: a sensação de desgaste generalizado sem causa aparente e dificuldades com a memória. No nível fisiológico, muitas mudanças ocorrem principalmente em termos do funcionamento das glândulas supra-renais: a medula diminui a sua produção de adrenalina e seu córtex produz mais corticosteróide podendo o sistema imunológico ficar afetado, o que aumenta a probabilidade da pessoa adoecer. Os jovens avaliados tinham, portanto, uma probabilidade de virem a adoecer devido ao gasto de energia envolvido para lidarem com os estressores do momento.

Considerando que o stress era maior durante o período de preparo para o vestibular, deduz-se que este é um estressor de grande porte para os jovens, o que leva ao questionamento da adequação de um sistema tão competitivo e estressante ao qual um jovem tem que se submeter para ter simplesmente o direito de estudar. Levanta-se a possibilidade também de que a habilidade de lidar com o stress e a ansiedade talvez seja um elemento importante para o sucesso em um vestibular tanto ou talvez até mais do que a habilidade acadêmica ou o conhecimento.

É importante salientar que na amostragem do presente estudo os sintomas mais freqüentes categorizados no ISS, para o sexo feminino, foram relacionados à "sensibilidade emotiva excessiva", seguido de "irritabilidade excessiva". Para o sexo masculino, os sintomas que prevaleceram foram "pensamento constante sobre um só assunto", seguido de "sensação de desgaste físico constante". Nota-se assim a predominância para estes grupos de sintomas psicológicos. Estes sintomas citados podem dificultar o acesso às habilidades acadêmicas necessárias aos estudantes, pois o aluno que inicia a universidade assume atividades rotineiras que envolvem alto desempenho e concentração de esforços voltados para uma rotina de estudos constantes e crescentes, podendo se tornar uma fonte de estímulos estressores.

Um dado que chamou a atenção foi o baixo relato do sintoma "diminuição da libido". A quase ausência desta queixa que é encontrada com freqüência em pessoas na fase de resistência ao stress, provavelmente se deve ao fato dos estudantes não desejarem relatar algo tão íntimo em uma situação de sala de aula. Talvez esta queixa pudesse ser detectada diferentemente se os dados fossem apurados dentro de um contexto clínico. Outra possibilidade a salientar é o fato de que em populações jovens, devido a intensidade hormonal, poderia efetivamente não haver uma diminuição da libido quando da presença de stress. Esta possibilidade requer estudos mais específicos.

O presente estudo torna-se relevante quando se verifica que há poucas pesquisas sobre stress, diferenças sexuais e vida acadêmica. Campos (1996) já havia ressaltado a importância de se implantar programas de apoio à pesquisa sobre stress acadêmico, seja na pré-escola, universidade ou 
pós-graduação. A necessidade da comunidade acadêmica acompanhar, monitorar e fornecer apoio a todos os seus componentes é visível e somente com sua realização é que muitos dos fatores que contribuem para o fracasso e a evasão escolar serão combatidos.

No presente estudo, além das diferenças em incidência de stress relacionadas ao momento acadêmico dos participantes, a diferença de sexo apresentou-se como outra variável importante e sistemática. Novos trabalhos devem investigar as fontes de stress em nossa cultura e seu efeito diferencial em homens e mulheres em diversas faixas etárias, principalmente aquelas ligadas a práticas parentais que possam estar gerando um stress maior em mulheres brasileiras.

\section{Referências}

Anthony, E. J. \& Cohler, B. J. (1992). The vulnerable child. New York: Guilford Press.

Arnett, J. J. (1999). Adolescent storm and stress reconsidered. American Psychologist, 54, 317-326.

Campos, L. F. L. (1996). Stress na Universidade. Em Pontifícia Universidade Católica de Campinas (Org.), Anais do I Simpósio sobre Stress e suas Implicações, (pp. 23-27). Campinas: PUC- Campinas.

Campos, L. F. L., Rocha, R. L., Campos, P. R. \& Oliveira, E. G. (1996). Estresse em estudantes universitários: Um estudo longitudinal. Psicologia Argumento, 14, 83-109.

Earls, F., Beardslee, W. \& Garisson, W. (1992). Correlates and predictors of competence in young children. Em E. J. Anthony \& B. J. Cohler (Orgs.), The vulnerable child (pp. 70-83). New York: Guilford Press.

Everly, G. S. (1989). A clinical guide to the treatment of the buman stress response. New York: Plenum Press.

Fisher, S. (1994). Stress in academic life. London, England: Open University. Gadzella, B. M., Ginther, D. W., Tomcala, M. \& Bryant, G. W. (1991). How business professionals view their stress. Psychological Reports, 68, 396-398.

Haslam, C., Stevens, R. \& Haslam, R. (1989). Eating habits and stress correlates in a female student population. Work \& Stress, 3, 327-334.

Knobel, M. (1985). A Síndrome da adolescência normal. Em A. Aberastury \& M. Knobel (Org.), Adolescência normal. (4ª ed.) (pp. 5-15). Porto Alegre: Artes Médicas. (Original publicado em 1970)

Langston, A. C. \& Cantor, N. (1989). Social anxiety and social constraint: When making friends is hard. Journal of Personality and Social Psychology, 56, 649661.
Lipp, M. E. N (2000). Crianças estressadas: Causas, sintomas e soluções. Campinas, SP: Papirus.

Lipp, M. E. N. \& Guevara, A. J. H. (1994). Validação empírica do Inventário de Sintomas de Stress (ISS). Estudos de Psicologia, 11, 43-49.

Lipp, M. E. N., Pereira, I. C., Floksztrumpf, C., Muniz, F. \& Ismael, S. C. (1996). Diferenças em nível de stress entre homens e mulheres na cidade de São Paulo. [Resumos]. Em Pontifícia Universidade Católica de Campinas (Org.), Anais do I Simpósio sobre Stress e suas Implicações. Campinas: PUCCampinas (p. 22).

Lipp, M. E. N. \& Rocha, J. C. (1994). Stress, hipertensão arterial e qualidade de vida. Campinas, SP: Papirus.

McWilliams, J. M. \& Schnorr, J. K. (1986). Tennesse Stress Scale-L: Work-Related Stress Inventory for Professionals. Arizona: Northern Arizon University Press.

Mulatu, M. S. (1995). Prevalence and risk factors of psychopathology in Ethiopian children. Journal AMACAD Child and Adolescence Psychiatry, 34, 19-25.

Schowalter, J. E. (1995). Desenvolvimento adolescente e normal. Em H. I. Kaplan \& B. J. Sadock (Orgs.), Tratado de Psiquiatria (Vol.3, pp. 2356-2362). Porto Alegre: Artes Médicas.

Scott, N. A. (1992). Chief student affairs officers: Stressors and strategies. Journal of College Student Development, 33, 108-116.

Seeman, M. V. (1997). Psychopatology in women and men: Focus on female hormones. American Journal of Psychiatry, 154, 1641-1647.

Selye, H. (1956). The stress of life. New York: McGraw-Hill. Short, K. H. \& Johnston, C. (1997). Stress, maternal distress, and children's adjustment following immigration: The buffering role of social support. Journal of Consulting and Clinical Psychology, 65, 494-503.

Spielberger, C. D. (1976). The nature and measurement of anxiety. Em C. D. Spielberger \& R. Diaz-Guerrero (Orgs.), Cross-cultural anxiety (pp. 47-64). Washington, DC: Hemisphere.

Tanganelli, M. S. L. \& Lipp, M. E. N. (1998). Sintomas de stress na rede pública de ensino. Estudos de Psicologia, 15, 17-27.

Tricoli, V. C. (1997). Sintomas de stress em escolares de $1^{a}$. a $4^{a}$. séries. Dissertação de Mestrado não-publicada, Curso de Pós-graduação em Psicologia, Pontifícia Universidade Católica de Campinas. Campinas, SP.

Vilela, M. V. (1996). Sintomas e fontes de stress em escolares de $1^{\text {a a a }} 4^{\mathrm{a}}$ séries. Em M. E. N. Lipp (Org.), Pesquisas sobre Stress no Brasil: Saúde, ocupacõos e grupos de risco (pp. 255-274). Campinas: Papirus.

Sobre as autoras

Sandra Leal Calais é Psicóloga, Doutora em Psicologia pela Pontifícia Universidade Católica de Campinas, Professora Assistente-Doutor do curso de Psicologia da Faculdade de Ciências, da Universidade Estadual Paulista "Júlio de Mesquita Filho", Unesp, Campus Bauru.

Lívia Márcia Batista de Andrade é Psicóloga, Mestre em Psicologia pela Pontifícia Universidade Católica de Campinas e Doutora pela mesma universidade, Professora da Faculdade de Psicologia Padre Anchieta (Jundiaí, SP).

Marilda Emmanuel Novaes Lipp é Ph.D. em Psicologia pela George Washington University (EUA), Professora Titular da Pontifícia Universidade Católica de Campinas e Diretora do Laboratório de Estudos Psicofisiológicos do Stress da mesma universidade. 